\title{
On the influence of thermal processes on the dynamics of a drill during deep hole machining
}

\author{
Leonid Kondratenko ${ }^{1}$ and Lubov Mironova, ${ }^{1, *}$ \\ ${ }^{1}$ Moscow Aviation Institute (State National Research University), Russia
}

\begin{abstract}
Deep drilling process has been studied and dynamic features of interaction the cutting part of the gun drill with the detail have been identified. Thermal processes of cutting and heat sinking in the interacting chains were analyzed: "heat source - workpiece"; "heat source - chips"; heat source - tool"; "heat source - cooling liquid". It has been noted that the vibrations lead to the loss of dimensional accuracy of the part fabrication as well as hole surface quality. Mathematical dependence of the longitudinal and torsional oscillations of the cutting drill bit is determined and the influence of heat flow pulsations on the friction coefficients and cutting force is revealed.
\end{abstract}

\section{Introduction}

During the recent years, studies of dynamic processes during deep drilling holes have been carried out [1-8]. This became due to higher demands of the machine-building industry for quality holes. In order to define forces at the cutting edges, commonly known equations are used usually that are results of approximation of experimental data, in which only cutting parameters: cutting depth, drill feed, machining sizes [4]. Such approach cannot be applied in deep drilling technology.

The work [3] is worth to be paid attention, where an approach is denoted to development of a common theory of the process in question. Nevertheless, it also reveals some omissions related to formation and removal of chips from the holes, relation between the longitudinal and torsional oscillations of the cutting part, deformation of the drill bit, etc $[5,9]$. The dynamics of the drilling process are strongly influenced by thermal processes that change the friction coefficients, etc. Therefore, the study of the influence of friction forces and thermal processes due to the interaction of the drill with metal during hole machining is an urgent task in the high-quality manufacture of work piece.

\section{Theoretical aspect}

In fig. 1 shows a drilling scheme for deep holes. Drilling process is complicated by the uneven rotation, elasticity of line, variable rotational moment and cutting force, precession

\footnotetext{
*Corresponding author: mironova_lub@mail.ru
} 
oscillation of axis of rotation and longitudinal oscillations of the cutting part. This leads to faceting, to significant deviations, to loss of dimensional accuracy and so on $[4,5]$.

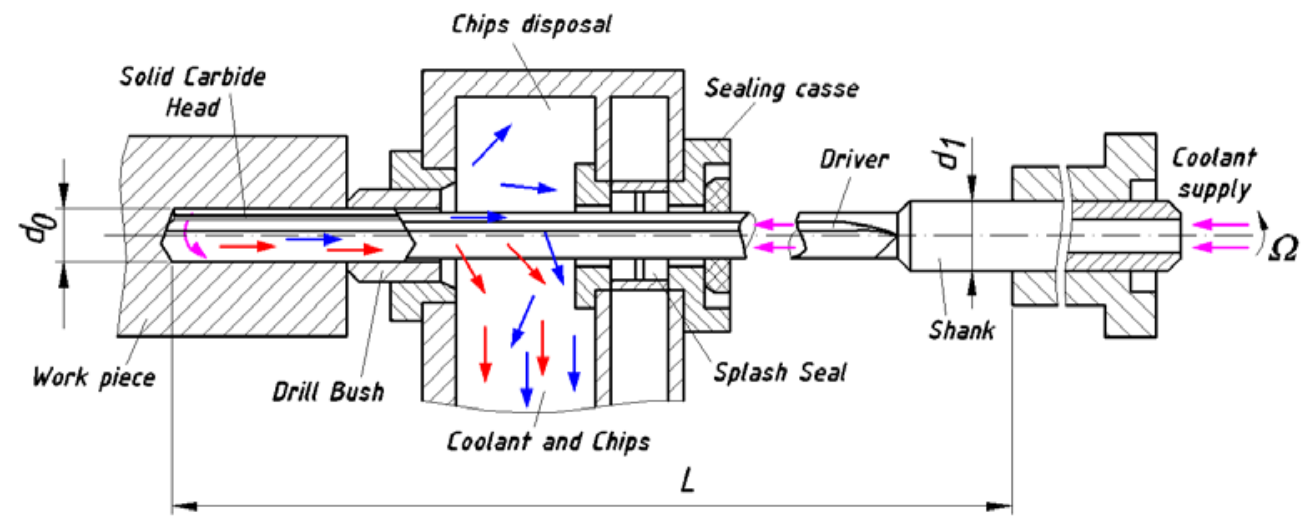

Fig. 1. Deep drilling

Thermal processes due to frictional force during the interaction of the cutting part of the tool with the part have been little studied.

In the essay [7] given a mathematical model of deep hole drilling process. The formalization is based on the integral equation describing the total moment acting on the cutting part of the gun drill, which allows us to give a qualitative assessment of the factors influencing the formation of the moment of resistance $M_{r}$. This equation has the form

$$
\begin{gathered}
M_{r}=M_{f}+\frac{1}{\cos \varphi_{1}} \int_{0}^{l} \psi_{f} \Phi r d r+\frac{1}{\cos \varphi} \int_{l}^{r_{d}} \psi_{f} \Phi r d r, \\
\Phi=\Phi^{\prime}+\Phi_{0}^{\prime}+\Phi_{0}^{\prime \prime}+\Phi_{0}^{\prime \prime \prime} .
\end{gathered}
$$

Where $M_{f t}$ is the friction moment of the tools, $\psi_{c}$ is a function of forces variation during cutting subject of the drill rotation speed; $\Phi^{\prime}, \Phi^{\prime}{ }_{0}, \Phi^{\prime \prime}{ }_{0}, \Phi^{\prime \prime \prime}{ }_{0}$ are functions depending on all specific cutting parameters and forces acting on the cutting part; $r_{d}$ is drill radius (Fig. 2); $r$ is actual radius of a layer: $\varphi, \varphi_{1}$ is inclination angles of the cutting edges; $l, r_{d}$ is integration limits, $l$ is border of the cutting edges transition $\left(l=r_{c}-m\right)$.

In general, this equation is applicable for any drill for single-side drilling.

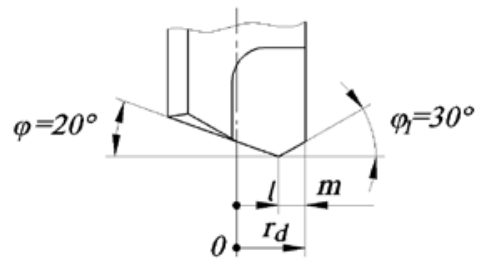

Fig. 2. Cutting part of the gun drill

Therefore, the power consumed for drilling can be defined by equation

$$
N_{\Sigma}=M_{r} \Omega
$$


where $\Omega$ is angular speed of the head rotation.

This power is spent on the deformation during the transformation of the cutting metal layer into chips, overcoming the friction forces on the front surface of the tool and the support elements of the spikelet, on overcoming the self-oscillating (vibrational) movements of the drill, and also on the heat losses going into the tool and carried away by both chips and coolant fluid.

It is obvious that the temperature in the cutting zone is significantly different from the temperature in the contact zone of the supporting elements of the cutting part.

The power consumed at these elements:

$$
N_{f}=M_{r} \Omega,
$$

Besides, the process of sliding of the support of the cutting part of the tool along the hole surface comes amid many effects. The list of some of them, as significant for mathematic formalization of the deep drilling process, is provided below:

1. Lubrication and heat removal from the contacting surfaces of the tool and removed layer of metal is provided by the cooling liquid, which comes into the radial gap between the cylindrical surfaces of the head and hole, Fig. 1.

2. The friction forces depend on the speed of mutual motion of surfaces and radial forces, acting onto the head. In this case, friction of different types can occur, like: fluid friction, boundary friction, dry friction, etc. In our opinion, friction losses will be different.

3. During the cutting process, contact interaction occurs between the cylindrical surface of the support element, of the liquid layer and the wall of the hole. We accept that this interaction system is an elastic system with its own dynamic characteristics, which under certain conditions can strongly influence the friction process.

4. If foreign particles (chips, metal particles, abrasive particles, etc.) fall between the supporting elements and wall of the hole, the friction process changes immediately.

These factors influence substantially the drilling process, and require consideration of a variable friction coefficient, which will define, in its turn, variation of the cutting forces. Thus, for scrutiny of the deep drilling process in details, it is necessary to take into account totality of the parameters and character of the friction forces. [5]

The power of the $N_{T}$ in the cutting zone is spent on heat transfer in different directions

$$
N_{T}=\sum_{i=1}^{k} \frac{\Delta T_{i}}{R_{i}} .
$$

Here $\Delta T_{i}$ is temperature drop in each heat transfer channel; $R_{i}$ is heat resistance in each $i$-th channel.

According to [5, 9], the heat resistance during the heat transfer without formation of any protective films can be found by

$$
R_{i}=\frac{\Delta_{i}}{f_{i} \cdot \lambda_{i}},
$$

where $\Delta_{i}$ is a distance of the heat wave or depth of the layer in question; $f_{i}$ is area of the contact; $\lambda_{i}$ is coefficient of heat conductivity of the channel.

Studies of temperature fields $[5,9,10]$ indicate that cutting results in at least three sources of heat during cutting:

- on the front surface of the tool; 
- on the surface of the part in close proximity of to the tip point contact;

- in removed chips.

Variable effects cause mechanical oscillations in the system "cutting part - spindle". After stronger oscillations, assessment of the dynamic aspects of the mechanical drive of the machine is a must.

If neglecting the spindle oscillations, the equation of rotation of the cutting part for the system in question can be written as follows [11]

$$
\Omega_{2}(s) \cdot\left[1+h_{k} \vartheta_{k}(s) s+J \vartheta_{k}(s) s^{2}\right]=\frac{\Omega_{1}(s)}{c h\left[\theta_{k}(s) l\right]}-M_{r}(s) \vartheta_{k}(s) s,
$$

where $\theta_{k}$ is operator coefficient of waves propagation; $l, r, W_{p 2}, G, \rho$ are length, radius, geometrical resistance moment of the drill bit cross-section, shear modulus and density of the drill bit metal, correspondingly; $J$ is flywheel moment of the cutting part inertia; $h_{k}$ is coefficient of the friction losses; $\Omega_{2}, \Omega_{1}$ is angular speed of the cutting part and spindle; $M_{r}$ is resistance moment; $s$ is complex variable.

In the formula (7), the following relations taken [11]

$$
\vartheta_{k}(s)=\vartheta_{k 0} Z_{k}(s) ; \vartheta_{k 0}=\frac{2 l r}{G W_{p 2}} ; Z_{k}(s)=\frac{\operatorname{th}\left[\theta_{k}(s) l\right]}{\theta_{k}(s) l}, \theta_{k}(s)= \pm s \sqrt{\frac{\rho}{G}} .
$$

Longitudinal oscillations of the cutting part can also be described by equation $[11,12]$

$$
\left(m_{2} \vartheta_{0} s^{2}+h \vartheta_{k 0} s+1\right) \cdot v_{2}(s)=v_{1}(t)-v_{0} s P(s),
$$

where $m_{2}, \vartheta_{0}, h$ are mass of the cutting part, longitudinal flexibility of the drill bit, coefficient of friction losses, correspondingly; $v_{2}, v_{0}$ are speed of longitudinal travel of the cutting part and spindle; $P$ is resistance force.

By substitution of $s=j \omega$, where $j$ is imaginary number and $\omega$ is angular speed of oscillations, it is possible, for the appropriate initial conditions, calculate and build various frequency characteristics which help to analyze dynamic effects of drilling.

In the process of oscillations of the moment resistance and angular velocity of rotation, the power consumption changes. This leads to a change in heat fluxes and all parameters during the cutting process. It is possible to take into account the above by applying the approach described in [3].

When drilling, the chip separation process occurs along the entire length of the cutting edge. Moreover, at each point there are different speeds, forces, parameters of the cutting blade. In this case, you should select each point of the cutting edge of the drill and determine for this point all the factors of influence (angular velocity of rotation, cutting force, moment of resistance, etc.). The resulting calculated data must be added using a special program that must take into account all cutting conditions.

From the theory of cutting [13] it is known that the parameters of the cutting process, the friction angle $\rho$ and the cleaving angle $\theta$, as well as the cutting force $P_{z}$, depend on temperature $T$. Following our reasoning, we will evaluate the factors of influence in the cutting process.

We accept that each i-th point of the cutting edge of the drill is described by a radius $r_{i}$. The geometric parameters of the drill are known. The thickness of the cut layer $a$ at each $i$ th point is determined by the formula 


$$
a=S_{z o} \sin \varphi
$$

where $S_{z o}$ is the feed per revolution for one cutting edge.

We assume that the friction function $\mu$ depends on the following variables: temperature $T$, normal stress occurring on the surface of the cutting edge $\sigma_{s}$, cutting speed $v_{c}$ and thickness of the removed layer $a$. Then the following relation holds:

$$
\mu=f\left(T, \sigma_{s}, v_{c}, a\right) .
$$

We expand equation (11) in a Taylor series. As a first approximation, we accept only a change in temperature. Using the first derivative, we obtain the following expression [3, 13]

$$
\mu=\mu_{0}+\frac{\partial \mu}{\partial T} \Delta T=\mu_{0}+A_{\mu} \exp \left(B_{\mu} T+C_{\mu} T^{2}\right)\left(B_{\mu}+2 C_{\mu} T\right) .
$$

Where: $A_{\mu}=0.5-0.6 ; B_{\mu}=0.069 ; C_{\mu}=-1.16 \cdot 10^{-5}[14]$.

The initial $\mu_{0}$ value is selected from the graph in Fig. 3 at the point where all the curves almost intersect, i.e. $\mu_{0}=1.1 ; T=200^{\circ} \mathrm{C}$.

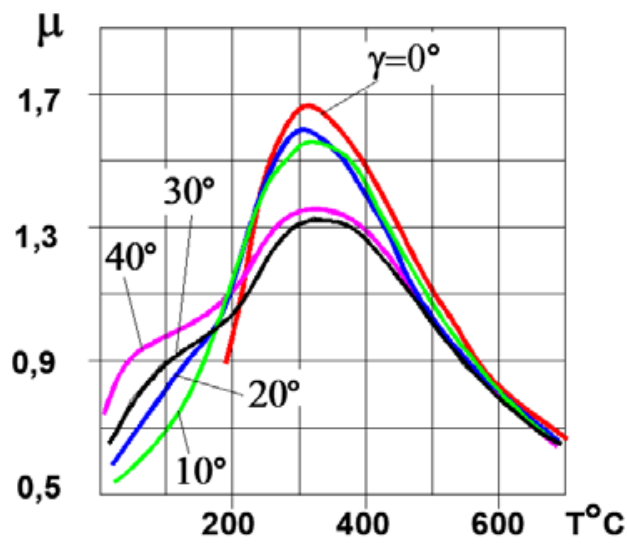

Fig. 3. Experimental dependences of the average coefficient of friction, calculated taking into account the average actual rake angle on the average temperature of contact of the cutter with the processed material with free cutting [13].

Shear stress are determined by the formula $[3,13]$

$$
\tau_{s}=\tau_{s 0}+\frac{\partial \tau_{s}}{\partial v_{c}} \Delta v_{c}+\frac{\partial \tau_{s}}{\partial T} \Delta T=\tau_{s 0}+\eta v_{c}^{\eta-1}(A \varepsilon+B) \Delta v_{c}-2 A_{t} T \Delta T
$$

Where $\tau_{s 0}$ is the initial value of the shear stress; $A, A_{1}, B$ are decomposition coefficients; $\eta$ is the order of the differential equation; $\varepsilon$ is the value characterizing the geometric parameters of the drill.

In the formula (13) the following relations

$$
\tau_{s 0}=0.9 \sigma_{B}[14] ; \varepsilon=\frac{\cos \gamma}{\sin \theta \cos (\theta-\gamma)}
$$


where $\sigma_{B}$ is the tensile strength of the material; $\theta$ is the shear angle; $\gamma$ is the rake angle of the drill.

Coefficients $A, A_{1}, B$ reflect the tendency to harden and are determined from [13]. So for example, for steel 35: $A=8 ; A_{1}=0 ; B=20 ; \eta=0.05$. For a drill with a rake angle of $\gamma=2^{\circ}$, $\theta=22^{\circ}[13]$.

The components of the cutting force are determined by the formulas

$$
P_{z}=\frac{\tau a b \sin (\delta+\rho)}{\sin (\delta+\rho+\theta) \sin \theta} ; N=\frac{\tau a b \cos \rho}{\sin (\delta+\rho+\theta) \sin \theta} .
$$

Where $P_{z}$ is the cutting force parallel to the movement of the cutting edge; $N$ is normal force; $b$ is the length of the cut layer; $\delta$ is cutting angle $(\delta=90-\gamma) ; \rho$ is the angle of friction $\left(\rho=48^{\circ}\right)$.

Using the method of successive approximation with a given accuracy of calculation

$$
m=\mu_{i}-\mu_{i-1},
$$

we determine the values of the coefficient of friction and other parameters.

Taking into account formulas (5), (6), (14), (15), we present the final formula that allows us to estimate the temperature change in the cutting zone

$$
\Delta T=\frac{P_{z}}{a b} \frac{10^{6}}{4,187 \rho_{c} 125}+\frac{P_{z} \cup_{c}\left(R_{2}+R_{3}\right)}{60} .
$$

Where $R_{2}, R_{3}$ are the thermal resistance of the second and third channels of the heat source; $\rho_{c}$ is the density of the chips. The calculations of the parameters $R_{2}$ and $R_{3}$ are given in detail in [3].

In accordance with the described methodology, a special computer program was developed. We present the results of calculations of the desired parameters with the following initial data: hole diameter is $16 \mathrm{~mm}$; cutting tool is a gun drill; the feed per revolution for one cutting edge $S_{z o}=0.05 \mathrm{~mm} / \mathrm{rev}$; the calculation accuracy is $\mathrm{m}=0.1$. The calculations were made for two cutting modes. The calculation results are shown in table 1.

Table 1. Design parameters

\begin{tabular}{|l|c|c|c|}
\hline \multicolumn{1}{|c|}{ Option Number } & $P_{z}, N$ & $T,{ }^{\circ} \mathrm{C}$ & $\mu$ \\
\hline First option: $v_{c}=60 \mathrm{~m} / \mathrm{min}$ & 1521 & 106 & 0.63 \\
\hline Second option: $v_{c}=40 \mathrm{~m} / \mathrm{min}$ & 1471 & 104 & 0.62 \\
\hline
\end{tabular}

\section{Conclusions}

These results show that thermal processes during deep drilling do not significantly affect the coefficient of friction. Therefore, in the calculations according to equations (7-9) the friction loss coefficients can be taken constant.

\section{References}

1. Messaond, C. Weis, J. Sound Vib, 326 (2009)

2. O. A. Kondratyuk, J. Equipment and tools for professionals, 6 (2005) 
3. L. A. Kondratenko, Vibrations and speed regulation methods of movement of technological objects (MRSU, Moscow, 2005)

4. G. I. Granovsky, V. G. Granovsky, Metal cutting (Vysshaya shkola, Moscow, 1985)

5. V. M. Terekhov, L. A. Kondratenko, L. I. Mironova, J. Engineering \& Automation Problems, 4 (2016)

6. L. Kondratenko, L. Mironova, V. Terekhov, J. Vibroengineering Procedia, 11 (2017)

7. V. M. Terekhov, L. A. Kondratenko, J. Of machinery manufacture and reliability, 1 (1999)

8. L. Mironova, L. Kondratenko, Mathematical modeling of the processing of holes on CNC machines, J. Materials Today: Proceedings (Online 14 Aug 2019)

9. V. Terekhov, A. Smirnov, L. Mironova, Thermal phenomena and dynamic features of deep holes fabrication for connections of heat exchange tubes, J. Materials Today: Proceedings (Online 31 July 2019)

10. N. Reznikov, L. A. Reznikov, Thermal processes in technological systems (Mashinostroenie, Moscow, 1990)

11. L. A. Kondratenko Calculation of velocity variations and stresses in machine assemblies and components (Sputnik, Moscow, 2008)

12. L. A. Kondratenko, L. I. Mironova, J. Engineering \& Automation Problems, 4 (2018)

13. M. I. Klushin, Metal cutting (Mashgiz, Moscow, 1959)

14. YU. P. Holmogorov, Optimization of hole machining processes (Mashinostroenie, Moscow, 1984) 